Pacific Journal of Mathematic

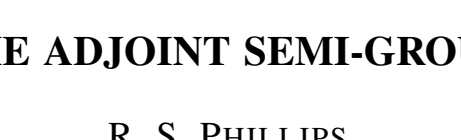




\title{
THE ADJOINT SEMI-GROUP
}

\author{
R. S. PHILLIPS
}

Introduction. The purpose of this paper is to develop a general theory for the adjoint semi-group of operators which fits into the framework of the present theory of semi-groups. To each semi-group of linear bounded operators $[T(s)]$ defined on a Banach space $\mathfrak{X}$ to itself and possessing suitable continuity properties, we shall assign an adjoint semi-group with like continuity properties, defined on an "adjoint" Banach space $\mathfrak{X}^{+}$which is in general a proper subspace of the adjoint space $\mathfrak{X}^{*}$. The usefulness of the adjoint semi-group has already been demonstrated by W. Feller $[3]$ in his treatise on the parabolic differential equation. ${ }^{1}$

In our theory of the adjoint semi-group, the choice of the subspace $\mathfrak{X}^{+} \subset \mathfrak{X}^{*}$ is decisive. We have been led to $\mathfrak{X}^{+}$by two independent considerations. In the first place $\mathfrak{X}^{+}$is the largest domain over which the ordinary adjoint $T^{*}(s)$ has suitable continuity properties. It should be noted, however, that a rather extensive theory of semi-groups has been developed by W. Feller [4] which has no such continuity requirements. The more compelling reason for our choice of $X^{+}$has to do with the infinitesimal generator. In most applications of the theory of semi-groups one starts with an infinitesimal generator $A$ and it is desired to establish the existence of a semi-group of operators generated by $A$. It is natural to expect the behavior of the semi-group operators $T(s)$ to be uniquely determined on the domain of $A$ (in symbols $\mathscr{P}(A)$ ); and since $T(s)$ is required to be bounded, there will exist a unique extension to the smallest closed subspace containing $\mathscr{S}(A)$, namely $\overline{S(A)}$. Further extensions are not uniquely determined by $A$ and should not be associated with the operator $A$. A reasonable approach to the adjoint semi-group would be to require that its infinitesimal generator be the adjoint $A^{*}$ of the infinitesimal generator $A$ of the original semi-group. In accordance with the above remarks, the proper domain for the adjoint semi-group

${ }^{1}$ It is remarkable that Feller actually obtained the entire adjoint semi-group without employing a precise notion for the adjoint to an unbounded operator such as the infinitesimal generator. For without this, the general formulation loses much of its significance.

Received August 30,1953. This paper was written under the sponsorship of the Office of Ordnance Research, U.S. Army, under Contract DA-04-495-ORD-406.

Pacific J. Math. 5 (1955), 269-283 


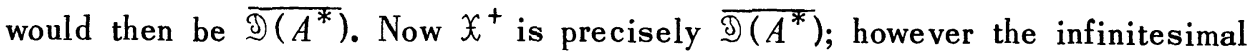
generator $A^{+}$of the adjoint semi-group turns out to be the maximal restriction of $A^{*}$ with domain and range in $\widehat{\mathscr{D (}\left(A^{*}\right)}=\mathfrak{X}^{+}$.

As in the ordinary theory of adjoint spaces, it is possible to develop an entire hierarchy of "adjoint" spaces for a given semi-group of operators. " However it can happen that the second "adjoint" is equal to the original space (under the natural mapping); in this case nothing new is achieved by going

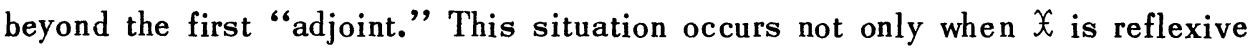
in the usual sense but, more generally, when the resolvent of $A$ is weakly compact (as in the case of most nonsingular problems of mathematical physics).

1. The adjoint transformation. We take $\chi$ and $\mathscr{V}$ to be Banach spaces over the real (or complex) scaler field. The transformation $y=T(x)$ is taken to be linear with domain $\mathscr{D} \subset X$ and range $\Re \subset \mathscr{V}$, and it is assumed that $\mathscr{D}$ is a linear

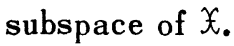

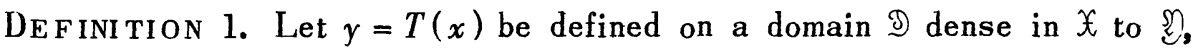
and let $\mathfrak{X}^{*}$ and $\mathfrak{Y}^{*}$ be the adjoint spaces to $\mathfrak{X}$ and $\mathscr{Y}$ respectively. The adjoint transformation $T^{*}$ of $T$ is defined as follows: Its domain $\mathscr{O}\left(T^{*}\right)$ consists of the set of all $y^{*} \in \mathbb{Z}^{*}$ for which there exists an $x^{*} \in X^{*}$ such that $y^{*}[T(x)]=x^{*}(x)$ for all $x \in D$; for such a $y^{*}$ we define $T^{*}\left(y^{*}\right)=x^{*}$.

It is clear that the density of $\mathscr{D}$ in $\mathcal{X}$ is required in order that $T^{*}$ be singlevalued. Further it is easy to show that $T^{*}$ is a closed linear transformation on $D\left(T^{*}\right)$ to $X^{*}$. On the other hand the second adjoint is not always well defined since $\mathscr{D}\left(T^{*}\right)$ is in general not dense in $\mathfrak{S}^{*}$. In this connection we have:

THE OREM 1.1. If $T$ is a closed linear transformation with domain $\mathcal{D}$ dense

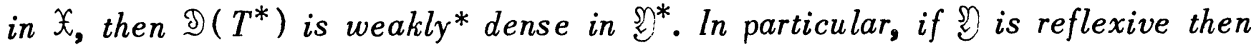
$\mathcal{D}\left(T^{*}\right)$ is strongly dense in $\mathfrak{S}^{*}$.

Proof. If $\supseteqq\left(T^{*}\right)$ were not weakly* dense in $\mathfrak{O}^{*}$, then the weak* closure of $\mathscr{D}\left(T^{*}\right)$ would be regularly closed [1] so that there would exist a $y_{0} \in \mathscr{Y}, y_{0} \neq 0$, such that $y^{*}\left(y_{0}\right)=0$ for all $y^{*} \in \mathscr{D}\left(T^{*}\right)$. Now $\left(0, y_{0}\right)$ does not belong to the graph $\&$ of $T$, and $\&$ is a closed linear subspace of $X \oplus \mathscr{S}$. Hence by a theorem

${ }^{2}$ For example if $X=C_{0}(-\infty, \infty)$, the space of continuous functions $f(\xi)$ on $(-\infty, \infty)$ such that $\lim |\xi| \rightarrow 0 f(\xi)=0$ and $\|f\|=\sup |f(\xi)|$, and if $A(f)=f^{\prime}, D(A)=[f ; f$ continuously differentiable, $f$ and $\left.f^{\prime} \in C_{0}\right]$, then $X^{+}=\mathrm{L}_{1}(-\infty, \infty),\left(X^{+}\right)^{+}=$space of all functions $f(\xi)$ uniformly continuous and bounded on $(-\infty, \infty)$ with $\|f\|=\sup |f(\xi)|$, and so on. 
due to H. Hahn [5, Theorem 2.9.4], there exists an

$$
\left(x_{0}^{*}, y_{0}^{*}\right) \in\left(\mathfrak{X} \oplus \mathfrak{C}^{*}\right)^{*}=\mathfrak{X}^{*} \oplus \mathfrak{I}^{*}
$$

such that

$$
x_{0}^{*}(x)+y_{0}^{*}[T(x)]=0 \text { for all } x \in \mathcal{D} \text { and } x_{0}^{*}(0)+y_{0}^{*}\left(y_{0}\right) \neq 0
$$

It follows that

$$
y_{0}^{*} \in \mathscr{D}\left(T^{*}\right), \quad T^{*}\left(y_{0}^{*}\right)=-x_{0}^{*}, \text { and yet } y_{0}^{*}\left(y_{0}\right) \neq 0,
$$

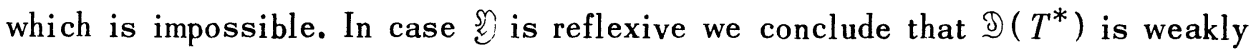
dense and hence strongly dense in $\mathfrak{S}^{*}$ (the latter conclusion follows from the above-mentioned Hahn theorem ).

We turn now to the relation between a transformation, its adjoint, and their inverses.

THEOREM 1.2. Let $T$ be a linear transformation with $\bar{\Phi}=\mathfrak{X}$. Then $\left(T^{*}\right)^{-1}$ exists if and only if $\bar{\Re}=?$. More generally, $\bar{\Re}$ consists of the set of all points $y$ such that $T^{*}\left(y^{*}\right)=0$ implies $y^{*}(y)=0$.

Proof. If $T^{*}\left(y_{0}^{*}\right)=0$, then

$$
\left[T^{*}\left(y_{0}^{*}\right)\right](x)=y_{0}^{*}[T(x)]=0
$$

for all $x \in \mathcal{D}$, and hence $y_{0}^{*}(\bar{\Re})=0$. In particular, $\bar{\Re}=\mathscr{N}$ implies that $y_{0}^{*}=0$, and hence that $T^{*}$ has an inverse. On the other hand if $y_{0} \notin \bar{\Re}$, then by the Hahn theorem there exists a functional $y_{0}^{*} \in \mathfrak{O}^{*}$ such that $y_{0}^{*}\left(y_{0}\right)=1$ and $y_{0}^{*}\left(\overline{\Re_{R}}\right)=0$. Thus $y_{0}^{*}[T(x)]=0$ for all $x \in \mathscr{D}$; it follows that $y_{0}^{*} \in \mathscr{D}\left(T^{*}\right)$ and $T^{*}\left(y_{0}^{*}\right)=0$; whereas $y_{0}^{*}\left(y_{0}\right) \neq 0$. In particular we see that if $\bar{\Re} \neq \mathcal{Y}$, then $T^{*}$ cannot have an inverse.

THEOREM 1.3. Let $T$ be a linear transformation with $\overline{\mathscr{D}}=\mathfrak{X}$. If $\Re\left(T^{*}\right)$ is weakly* dense in $\mathfrak{X}^{*}$, then $T$ has an inverse.

Proof. Suppose that $T$ has no inverse; then there is an $x_{0} \neq 0$ such that $T\left(x_{0}\right)=0$. Consequently

$$
\left[T^{*}\left(y^{*}\right)\right]\left(x_{0}\right)=y^{*}\left[T\left(x_{0}\right)\right]=0
$$

for all $y^{*} \in \mathscr{D}\left(T^{*}\right)$, and this shows that the weak* closure of $\Re\left(T^{*}\right)$ is a proper 
subspace of $\mathfrak{X}^{*}$, contrary to assumption.

THEOREM 1.4. Let $T$ be a linear transformation with an inverse and such that $\overline{\mathscr{D}}=\mathfrak{X}$ and $\bar{\Re}=\mathfrak{Y}$. Then $\left(T^{*}\right)^{-1}=\left(T^{-1}\right)^{*}$; further $T^{-1}$ is bounded if and only if $\left(T^{*}\right)^{-1}$ is bounded on $X^{*}$.

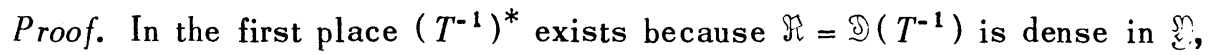
and $\left(T^{*}\right)^{-1}$ exists by Theorem 1.2. If $y \in \Re$ and $y^{*} \in \mathcal{O}\left(T^{*}\right)$, then

$$
y^{*}(y)=y^{*}\left\{T\left[T^{-1}(y)\right]\right\}=\left[T^{*}\left(y^{*}\right)\right]\left[T^{-1}(y)\right]
$$

This implies that $\Re\left(T^{*}\right) \subset \mathscr{D}\left[\left(T^{-1}\right)^{*}\right]$ and

$$
\left(T^{-1}\right)^{*}\left[T^{*}\left(y^{*}\right)\right]=y^{*}
$$

for all $y^{*} \in \mathscr{D}\left(T^{*}\right)$. Thus $\left(T^{-1}\right)^{*}$ is an extension of $\left(T^{*}\right)^{-1}$. On the other hand if $x \in \vartheta$, then

$$
x^{*}(x)=x^{*}\left\{T^{-1}[T(x)]\right\}=\left[\left(T^{-1}\right)^{*}\left(x^{*}\right)\right][T(x)],
$$

for all $x^{*} \in D\left[\left(T^{-1}\right)^{*}\right]$. It follows that $\Re\left(T^{*}\right) \supset \supset\left[\left(T^{-1}\right)^{*}\right]$. Therefore

$$
\supseteqq\left[\left(T^{-1}\right)^{*}\right]=\Re\left(T^{*}\right)=\supseteqq\left[\left(T^{*}\right)^{-1}\right] \text {, }
$$

and hence $\left(T^{-1}\right)^{*}=\left(T^{*}\right)^{-1}$. If, in addition, $T^{-1}$ is bounded, then it is clear that $\left(T^{-1}\right)^{*}$ is also bounded. Conversely if $\left(T^{*}\right)^{-1}$ is bounded on $\mathfrak{X}^{*}$, then for all $x \in \Re$ and $x^{*} \in \mathfrak{X}^{*}$ we have

$$
\left|x^{*}\left[T^{-1}(x)\right]\right|=\left|\left[\left(T^{-1}\right)^{*}\left(x^{*}\right)\right](x)\right| \leq\left\|\left(T^{*}\right)^{-1}\right\|\left\|x^{*}\right\|\|x\|
$$

It follows that $T^{-1}$ is bounded.

If $T$ is a linear operator with both domain and range in $X, \bar{D}=X$, then the adjoint transformation $T^{*}$ has its domain and range in $\mathfrak{X}^{*}$. It is easy to show for an arbitrary bounded operator $B$ on $X$ to itself, that

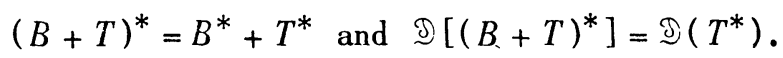

We are especially interested in the combination $\lambda-T$, where $I$ is the identity operator and $\lambda$ is a real (or complex) number. If $\lambda-T$ has a bounded inverse with domain dense in $\chi$, then $\lambda$ is said to belong to $\rho(T)$, the resolvent set of $T$, and 


$$
(\lambda I-T)^{-1} \equiv R(\lambda ; T)
$$

is called the resolvent of $T$.

THE OREM 1.5. If $T$ is a linear operator with $\bar{\Im}=\mathfrak{X}$ and $\Re \subset X$, then

$$
\rho(T)=\rho\left(T^{*}\right) \text { and }[R(\lambda ; T)]^{*}=R\left(\lambda ; T^{*}\right) .
$$

Proof. If $\lambda \in \rho(T)$, then, according to Theorem 1.4, $\lambda \in \rho\left(T^{*}\right)$ and

$$
[R(\lambda ; T)]^{*}=R\left(\lambda ; T^{*}\right) .
$$

On the other hand if $\lambda \in \rho\left(T^{*}\right)$, then Theorem 1.3 shows that $T$ has an inverse, Theorem 1.2 shows that $\bar{\Re}=\mathfrak{X}$, and Theorem 1.4 then implies that $\lambda \in \rho(T)$.

2. The adjoint semi-group. We now apply the previous results to semi-groups of linear bounded operators ( cf. $[5]$ ). Let $(\&(X)$ be the Banach algebra of endomorphism of $\mathfrak{X}$, and let $[T(s)]$ be a one-parameter family of operators in $\mathbb{E}(\mathfrak{X})$ defined for $s \in[0, \infty)$ and satisfying:

(i) $T\left(s_{1}+s_{2}\right)=T\left(s_{1}\right) T\left(s_{2}\right)$ for all $s_{1}, s_{2} \geq 0, T(0)=l$;

(ii) for each $x \in \mathfrak{X}, T(s) x$ is continuous for $s>0$;

(iii) $\int_{0}^{1}\|T(\sigma) x\| d \sigma<\infty$ for each $x \in \mathfrak{X}$.

If $T$ satisfies the additional condition

(iv) $\lim _{\lambda \rightarrow \infty} \lambda \int_{0}^{\infty} \exp (-\lambda \sigma) T(\sigma) x d \sigma=x$ for each $x \in \mathfrak{X}$, then $T(s)$ is said to be of class $(0, A)$. If, instead of (iv), $T(s)$ satisfies the stronger condition

$$
\text { (v) } \lim _{\mathcal{T} \rightarrow 0} \tau^{-1} \int_{0}^{\mathcal{T}} T(\sigma) x d \sigma=x \text { for each } x \in \mathfrak{X},
$$

then $T(s)$ is said to be of class $(0, C)$. Finally if $T(s)$ satisfies (i), (ii), (iii), and the still stronger continuity condition

(vi) $\lim _{s_{-} \rightarrow 0} T(s) x=x$ for each $x \in \mathfrak{X}$,

then $T(s)$ is said to be of class $C$.

The domain $\mathscr{S}(A)$ of the infinitesimal generator $A$ is the set of elements $x$ for which 


$$
\lim _{\tau \rightarrow 0} \tau^{-1}[T(\tau)-I] x
$$

exists, and this limit is defined to be $A x$. It follows from (iv) (and hence ( $v$ ) or (vi)) that $\mathscr{D}(A)$ is dense in $\mathscr{X}$ ( $\mathrm{cf}$. [5, Theorem 9.3.1]). We have previously shown [6] that $A$ is closed if and only if $T(s)$ is of class $(0, C)$. However, even when $T(s)$ is of class $(0, A)$, the infinitesimal generator has a smallest closed extension, called the complete infinitesimal generator (c.i.g.) and denoted by $\bar{A}$. For each $x_{0} \in \mathscr{D}(\bar{A})$ there is a sequence $\left\{x_{n}\right\} \subset \mathscr{D}(A)$ such that $x_{n} \longrightarrow x_{0}$ and $A x_{n} \rightarrow \bar{A} x_{0}$. It follows that $R(\lambda ; \bar{A})$ is an extension of $R(\lambda ; A)$, that $\rho(A)=\rho(\bar{A})$, that $A^{*}=(\bar{A})^{*}$, and that

$$
[R(\lambda ; A)]^{*}=[R(\lambda ; \bar{A})]^{*} .^{3}
$$

It can be shown that

$$
\omega_{0}=\inf _{s>0} \log \|T(s)\| / s=\lim _{s \rightarrow \infty} \log \|T(s)\| / s .
$$

Eacl. $\lambda>\omega_{0}$ belongs to the resolvent set for $\bar{A}$, and the resolvent is given by

$$
R(\lambda ; \bar{A}) x=\int_{0}^{\infty} \exp (-\lambda \sigma) T(\sigma) x d \sigma
$$

see [6].

Definition 2.1. The semi-group $T(s)$ is said to be of class $(0, A)^{*}$, $(0, C)^{*}$, or $C^{*}$ if it is of class $(0, A),(0, C)$, or $C$, respectively, and if in addition $\left\|T^{*}(s) x^{*}\right\|, 0 \leq s \leq 1$, is majorized by integrable function for each $x^{*} \in X^{*} \cdot{ }^{4}$

Definition 2.2. Let $T(s)$ be a semi-group of class $(0, A)$ with infinitesimal generator $A$. We define the adjoint semi-group to be the restriction of $T^{*}(s)$ to $\mathfrak{X}^{+}=\overline{\mathscr{S}\left(A^{*}\right)}$ and denote it by $T^{+}(s)$. We denote the infinitesimal generator of $T^{+}(s)$ by $A^{+}$.

${ }^{3}$ For $\lambda \in \rho(A)$, the resolvent $R(\lambda ; A)$ has a unique bounded linear extension $R(\lambda$; $A)_{1}$ on $\mathcal{X}$. If $\left\{x_{n}\right\} \subset \mathcal{O}(A), x_{n} \longrightarrow x_{0} \in \mathcal{D}(\bar{A})$, and $A x_{n} \rightarrow \bar{A} x_{0}$, then $R(\lambda ; A)(\lambda I-$ $A) x_{n}=x_{n}$ implies that $R(\lambda ; A)_{1}(\lambda I-\bar{A}) x_{0}=x_{0}$. Likewise for $\left\{y_{n}\right\} \subset \Re(\lambda I-A)$ and $y_{n} \longrightarrow y_{0}$, the relation $(\lambda I-A) R(\lambda ; A) y_{n}=y_{n}$ implies that $(\lambda I-\bar{A}) R(\lambda ; A)_{1} y_{0}=y_{0}$. It follows that $R(\lambda ; \bar{A})$ exists and is identical with $R(\lambda ; A)_{1}$. This shows that $\rho(A) C$ $\rho(\bar{A})$. A similar argument can be used to prove $A^{*}=\bar{A}^{*}$, and the last relation is obvious.

${ }^{4}$ This condition is automatically satisfied if $\int_{0}^{1}\|T(\sigma)\| d \sigma<\infty$ or if $T(s)$ if of class $C$. 
THe OREM 2.1. If $T(s)$ is a semi-group of class $(0, A)^{*},(0, C)^{*}$, or $C^{*}$, then the adjoint semi-group is of class $(0, A),(0, C)$ or $C$, respectively. The c.i.g. $\bar{A}^{+}$is the largest restriction of $A^{*}$ with domain and range in $\mathfrak{X}^{+}$.

Proof. According to Theorem 1.5,

$$
R\left(\lambda ; A^{*}\right)=R\left(\lambda ; \bar{A}^{*}\right)=R^{*}(\lambda ; A)
$$

and hence $\mathscr{D}\left(A^{*}\right)$ is simply the range of $R^{*}(\lambda ; A)$. For $\lambda>\omega_{0}, R^{*}(\lambda ; A)$ can be expressed by means of a Dunford integral $[2]$ as

$$
R^{*}(\lambda ; A) x^{*}=\int_{0}^{\infty} \exp (-\lambda \sigma) T^{*}(\sigma) x^{*} d \sigma
$$

It is clear from this that

$$
T^{*}(s) R^{*}(\lambda ; A)=R^{*}(\lambda ; A) T^{*}(s),
$$

so that $T^{*}(s)$ takes $\mathscr{D}\left(A^{*}\right)$ into $\mathscr{D}\left(A^{*}\right)$. Since $T^{*}(s)$ is bounded, it follows that $T^{*}(s)\left(X^{+}\right) \subset \mathfrak{X}^{+}$; that is, $T^{+}(s) \in \mathscr{E}\left(\mathfrak{X}^{+}\right)$. It is obvious that $T^{*}(s)$ and hence $T^{+}(s)$ satisfies (i).

In order to establish continuity we first note that

$$
\begin{aligned}
{\left[T^{*}(\tau)-I^{*}\right] R^{*}(\lambda ; A) x^{*}=} & {[\exp (\lambda \tau)-1] \int_{0}^{\infty} \exp (-\lambda \sigma) T^{*}(\sigma) x^{*} d \sigma } \\
& -\exp (\lambda \tau) \int_{0}^{\tau} \exp (-\lambda \sigma) T^{*}(\sigma) x^{*} d \sigma
\end{aligned}
$$

The first term in the right member is simply $[\exp (\lambda \tau)-1] R^{*}(\lambda ; A) x^{*}$, and it clearly converges to zero with $\tau$; further the assumption that $\left\|T^{*}(\sigma) x^{*}\right\|$ is majorized by a function in $L_{1}(0,1)$ implies that the second term also goes to zero with $\tau$. Thus

$$
\lim _{s \rightarrow 0} T^{*}(s) y^{*}=y^{*}
$$

for all $y^{*} \in \mathscr{D}\left(A^{*}\right)$. It follows from this ( $\mathrm{cf}$. [5, Theorem 9.4.1]) that $T^{*}(s) y^{*}$ is strongly continuous for $s \geq 0, y^{*} \in \mathfrak{O}\left(A^{*}\right)$. Further since $\left\|T^{*}(s)\right\|=$ $\|T(s)\|$ is uniformly bounded in each interval of the form $(\delta, 1 / \delta)$, we see that $T^{*}(s) x^{*}$ is strongly continuous for $s>0$ and all $x^{*} \in \mathfrak{X}^{+}$. Thus $T^{+}(s)$ satisfies (i), (ii), and (iii). Again, for each $x^{*} \in \mathcal{D}\left(A^{*}\right)$, 


$$
T^{+}(s) x^{*} \longrightarrow x^{*} \text { as } s \longrightarrow 0
$$

and $a$ fortiori

$$
\tau^{-1} \int_{0}^{\tau} T^{*}(\sigma) x^{*} d \sigma \longrightarrow x^{*} \text { as } \tau \longrightarrow 0
$$

and

$$
\lambda R^{*}(\lambda ; A) x^{*} \longrightarrow x^{*} \text { as } \lambda \longrightarrow \infty \text {. }
$$

Now if $T(s)$ is of class $C$, then $\left\|T^{*}(s)\right\|=O(1)$; if $T(s)$ is of class $(0, C)$ then

$$
\left\|\left[\tau^{-1} \int_{0}^{\tau} T(\sigma) d \sigma\right]^{*}\right\|=O(1)
$$

and if $T(s)$ is of class $(0, A)$ then $\left\|\lambda R^{*}(\lambda ; A)\right\|=O(1)$. It now follows from the Banach-Steinhaus theorem that $T^{+}(s)$ will satisfy (vi), (v), or (iv) with $T(s)$.

Finally, the c.i.g. $\bar{A}^{+}$of $T^{+}(s)$ is determined by its resolvent (cf. [6]), which for $\lambda>\omega_{0}$ can be expressed by the Bochner integral

$$
R\left(\lambda ; \overline{A^{+}}\right) x^{*}=\int_{0}^{\infty} \exp (-\lambda \sigma) T^{+}(\sigma) x^{*} d \sigma \quad\left(x^{*} \in \mathfrak{X}^{+}\right) .
$$

According to formula $(2.3)$ this is simply the restriction of $R\left(\lambda ; A^{*}\right)$ to $X^{+}$; thus $\overline{A^{+}}$is a restriction of $A^{*}$. Now if $x^{*} \in \mathscr{S}\left(A^{*}\right)$ and $A^{*}\left(x^{*}\right) \in X^{+}$, then $\left(\lambda I^{*}-A^{*}\right) x^{*} \in X^{+}$and hence

$$
R\left(\lambda ; A^{*}\right)\left(\lambda I^{*}-A^{*}\right) x^{*}=x^{*} \in \mathscr{I}\left(\overline{A^{+}}\right) .
$$

Conversely if $x^{*} \in \mathscr{D}\left(\overline{A^{+}}\right)$, then $x^{*} \in \mathscr{D}\left(A^{*}\right)$ and $A^{*} x^{*}=\overline{A^{+}} x^{*} \in X^{+}$. In other words, $\bar{A}^{+}$is the maximal restriction of $A^{*}$ which maps $\mathfrak{X}^{+}$into $\mathfrak{X}^{+}$. This concludes the proof.

COROLla RY. If $\lambda \in \rho(\bar{A})$, then $\lambda \in \rho\left(\overline{A^{+}}\right)$and $R\left(\lambda ; \overline{A^{+}}\right)$equals the restriction of $R\left(\lambda ; A^{*}\right)$ to $\chi^{+}$.

Proof. If $\lambda \in \rho(A)$, then $R\left(\lambda ; A^{*}\right)$ exists. Let $R\left(\lambda ; A^{*}\right)_{0}$ be the restriction of $R\left(\lambda ; A^{*}\right)$ to $\mathfrak{X}^{+}$. For $x^{*} \in \mathfrak{D}\left(\overline{A^{+}}\right)$, we have

$$
\left(\lambda I^{+}-\overline{A^{+}}\right) x^{*}=\left(\lambda l^{*}-A^{*}\right) x^{*}
$$


and hence $R\left(\lambda ; A^{*}\right)_{0}$ is a left inverse for $\lambda I^{+}-\overline{A^{+}}$. On the other hand if $x^{*} \in X^{+}$, then

$$
\left(\lambda I^{*}-A^{*}\right) R\left(\lambda ; A^{*}\right)_{0} x^{*}=x^{*} .
$$

Since $R\left(\lambda ; A^{*}\right)_{0} x^{*} \in \mathscr{D}\left(A^{*}\right) \subset \mathfrak{X}^{+}$we also have $A^{*} R\left(\lambda ; A^{*}\right)_{0} x^{*} \in \mathfrak{X}^{+}$and hence by the above theorem $R\left(\lambda ; A^{*}\right)_{0} x^{*} \in \mathscr{O}\left(\overline{A^{+}}\right)$. It follows that $R\left(\lambda ; A^{*}\right)_{0}$ is also the right inverse for $\lambda I^{+}-\overline{A^{+}}$so that $\lambda \in \rho\left(\overline{A^{+}}\right)$.

A converse to the above corollary is obtained in Theorem 3.2 where it is shown that $\rho(\bar{A})=\rho\left(\overline{A^{+}}\right)$.

COROLla RY. If $\mathfrak{X}$ is reflexive, then $\mathfrak{X}^{+}=\mathfrak{X}^{*}$.

Proof. If $X$ is reflexive, then, according to Theorem 1.1, $\supseteq\left(A^{*}\right)$ is dense in $\mathfrak{X}^{*}$. Hence $\mathfrak{X}^{+}=\overline{D\left(A^{*}\right)}=\mathfrak{X}^{*}$.

We conclude this section with two other characterizations of $\mathfrak{X}^{+}$.

THEORE м 2.2. For a semi-group $T(s)$ of class $(0, A)^{*}$, let

$$
\Gamma=\left[x^{*} ; T^{*}(s) x^{*} \longrightarrow x^{*} \text { as } s \longrightarrow 0\right] \text {. }
$$

Then $\mathfrak{X}^{+}=\bar{\Gamma}$.

Proof. It is clear that $\mathscr{D}\left(A^{*}\right) \subset \Gamma$; and since $\mathscr{D}\left(A^{*}\right)$ is dense in $\mathfrak{X}^{+}$, we have $X^{+} \subset \bar{\Gamma}$. On the other hand if $x^{*} \in \Gamma$, then a direct calculation shows that

$$
\lambda R\left(\lambda ; A^{*}\right) x^{*}=\lambda \int_{0}^{\infty} \exp (-\lambda \sigma) T^{*}(\sigma) x^{*} d \sigma \rightarrow x^{*} \quad \text { as } \lambda \longrightarrow \infty .
$$

Consequently $x^{*} \in \overline{\mathscr{D}\left(A^{*}\right)}=\mathfrak{X}^{+}$.

THE OREM 2.3. For a semi-group $T(s)$ of class $(0, A)^{*}$ let

$$
\Gamma_{0}=\left[y_{\alpha \beta}^{*} ; y_{\alpha \beta}^{*}=\int_{\alpha}^{\beta} T^{*}(\sigma) x^{*} d \sigma, x^{*} \in \mathfrak{X}^{*}, 0 \leq \alpha<\beta\right] .
$$

Then $\mathfrak{X}^{+}=\bar{\Gamma}_{0}$.

Proof. An easy calculation shows that $\Gamma_{0} \subset \Gamma$. On the other hand if $x^{*} \in \Gamma$ then

$$
\tau^{-1} \int_{0}^{\tau} T^{*}(\sigma) x^{*} d \sigma \longrightarrow x^{*}
$$


and belongs to $\Gamma_{0}$; thus $\bar{\Gamma}_{0} \supset \Gamma$ and therefore $\bar{\Gamma}_{0}=\bar{\Gamma}=\mathfrak{X}^{+}$.

3. The adjoint space. We shall call $\mathfrak{X}^{+}$the adjoint space to $\mathfrak{X}$ relative to the semi-group $[T(s)]$, or simply, the adjoint space; and we shall denote the generic element of $\mathfrak{X}^{+}$by $x^{+}$. To avoid confusion we shall hereafter refer to $X^{*}$ as the full adjoint space. This section is devoted to a study of the hierarchy of adjoint spaces which arise from a given semi-group of operators of class $(0, A)^{*}$.

It will be observed that whereas

$$
\left\|x^{*}\right\|=\sup \left[\left|x^{+}(x)\right| ;\|x\| \leq 1, x \in \mathfrak{X}\right],
$$

it is not in general true that $\|x\|$ can be obtained in like manner as

$$
\|x\|^{\prime}=\sup \left[\left|x^{+}(x)\right| ;\left\|x^{+}\right\| \leq 1, x^{+} \in \mathfrak{X}^{+}\right] .
$$

All that can be asserted here is that $\|x\|^{\prime} \leq\|x\|$. If $\mathfrak{X}^{+}$is equal to the full adjoint space, then it is clear that $\|x\|^{\prime}=\|x\|$. This occurs when $\chi$ is reflexive or when $A$ is bounded. In any case we see that the function $\|x\|^{\prime}$ satisfies the postulates of a pseudo-norm. However, more is true:

THEOREM 3.1. The norm $\|x\|^{\prime}$ defines an equivalent topology for $\mathfrak{X}$; in fact, there exists an $m>0$ such that

$$
\|x\| \geq\|x\|^{\prime} \geq m\|x\|
$$

for all $x \in \mathfrak{X}$. In particular if

$$
\liminf _{\lambda \rightarrow \infty}\|\lambda R(\lambda ; \bar{A})\|=1,
$$

then $\|x\| \equiv\|x\|^{\prime}$.

Proof. For a fixed $x \in \mathfrak{X}$ there exists an $x^{*} \in \mathfrak{X}^{*},\left\|x^{*}\right\|=1$, such that $x^{*}(x)=\|x\|$. It follows from (iv) that

$$
\left[\lambda R^{*}(\lambda ; \bar{A}) x^{*}\right](x)=x^{*}[\lambda R(\lambda ; \bar{A}) x] \rightarrow x^{*}(x) \quad \text { as } \lambda \rightarrow \infty,
$$

and from (iv) together with the uniform boundedness theorem that

$$
\varliminf_{\lambda \rightarrow \infty}\|\lambda R(\lambda ; \bar{A})\|=M<\infty .
$$


Consequently, given $\epsilon>0$, there is a $\lambda_{\epsilon}$ with

$$
\left\|\lambda_{\epsilon} R^{*}\left(\lambda_{\epsilon} ; \bar{A}\right)\right\| \leq M+\epsilon \text { and }\left|\left[\lambda_{\epsilon} R^{*}\left(\lambda_{\epsilon} ; \bar{A}\right) x^{*}\right](x)-\|x\|\right|<\epsilon .
$$

Now

$$
y_{\epsilon}^{*} \equiv \lambda_{\epsilon} R^{*}\left(\lambda_{\epsilon} ; A\right) x^{*} \in \mathfrak{X}^{+} \text {and }\left\|y_{\epsilon}^{*}\right\| \leq M+\epsilon \text {. }
$$

Hence

$$
\frac{\left|y_{\epsilon}^{*}(x)\right|}{\left\|y_{\epsilon}^{*}\right\|} \geq \frac{\|x\|-\epsilon}{M+\epsilon}
$$

and since $\epsilon$ is arbitrary this gives the desired result with $m=1 / M$. In particular if $M=1$, then $\|x\|=\|x\|$ :

THEOREM 3.2. If $[T(s)]$ is a semi-group of operators of class $(0, A)^{*}$, then $\rho(\bar{A})=\rho\left(\overline{A^{+}}\right)$.

Proof. We have already shown in the first corollary to Theorem 2.1 that $\rho(\bar{A}) \subset \rho\left(\overline{A^{+}}\right)$. If $\lambda \in \rho\left(\overline{A^{+}}\right)$, then

$$
\Re\left(\lambda I^{*}-\bar{A}^{*}\right) \supset \Re\left(\lambda I^{+}-\overline{A^{+}}\right)=X^{+} .
$$

Since, by Theorem 1.1, $\left(\bar{A}^{*}\right) \subset \mathfrak{X}^{+}$is weakly* dense in $\mathfrak{X}^{*}$, the same is true of $\Re\left(\lambda I^{*}-\bar{A}^{*}\right)$. It now follows from Theorem 1.3 that $\lambda I-\bar{A}$ has an inverse. Further, if

$$
\left(\lambda I^{*}-\bar{A}^{*}\right) x_{0}^{*}=0
$$

then $x_{0}^{*} \in \mathscr{S}\left(\bar{A}^{*}\right)$ and $\bar{A}^{*} x_{0}^{*} \in \mathscr{P}\left(\bar{A}^{*}\right) \subset X^{+}$, so that $x_{0}^{*} \in \mathscr{S}\left(\overline{A^{+}}\right)$. Since $\overline{A^{+}}$is a restriction of $\bar{A}^{*}$, this implies that $\left(\lambda I^{+}-\bar{A}^{+}\right) x_{0}^{*}=0$ and hence that $x_{0}^{*}=0$. Theorem 1.2 now asserts that $\Re(\lambda I-\bar{A})$ is dense in $\mathfrak{X}$. Finally for $x \in \Re(\lambda I-\bar{A})$ we have

$$
\begin{aligned}
\left\|(\lambda I-\bar{A})^{-1} x\right\| \leq & m^{-1}\left\|(\lambda I-\bar{A})^{-1} x\right\|^{\prime} \\
& =m^{-1} \sup \left[\left|x^{+}\left[(\lambda I-\bar{A})^{-1} x\right]\right| ;\left\|x^{+}\right\| \leq 1, x^{+} \in X^{+}\right] \\
& \leq m^{-1}\left\|R\left(\lambda ; \overline{A^{+}}\right)\right\|\|x\| ;
\end{aligned}
$$

and this shows that $(\lambda l-\bar{A})^{-1}$ is bounded. It follows that $\lambda \in \rho(\bar{A})$. 
We see from the above theorem that $\overline{A^{+}}$has the same resolvent set as $\bar{A}^{*}$ ( and $\bar{A}$ ) in spite of the fact that it is a restriction of $\bar{A}^{*}$.

Renorming $X$ by $\|x\|^{\prime}$ has no effect on our determination of $\mathfrak{X}^{+}$; in fact, even the norm of the elements of $X^{+}$remains the same. For

$$
\|x\|^{\prime} \leq\|x\| \text { and }\left|x^{+}(x)\right| \leq\left\|x^{+}\right\|\|x\|^{\prime}
$$

imply that

$$
\left\|x^{+}\right\| \leq \sup \left[\left|x^{+}(x)\right| ;\|x\|^{\prime} \leq 1, x \in X\right] \leq\left\|x^{+}\right\|
$$

Nevertheless, when we deal with the second adjoint space relative to a given semi-group $[T(s)]$, a slight advantage is obtained by renorming $\chi$ in this way.

THE OREM 3.3. Suppose that both $[T(s)]$ and $\left[T^{+}(s)\right]$ are of class $(0, A)^{*}$, and let the norm of $X$ be given by $\|x\|^{\prime}$. Then $X$ can be embedded in $\mathfrak{X}^{++}$by means of the natural mapping.

Proof. Each $x_{0} \in \mathfrak{X}$ defines a unique bounded linear functional $F_{0} \in\left(\mathfrak{X}^{+}\right)^{*}$, namely $F_{0}\left(x^{+}\right)=x^{+}\left(x_{0}\right)$. Further,

$$
\left\|F_{0}\right\|=\sup \left[\left|F_{0}\left(x^{+}\right)\right|=\left|x^{+}\left(x_{0}\right)\right| ;\left\|x^{+}\right\| \leq 1, x^{+} \in X^{+}\right]=\left\|x_{0}\right\|^{\prime} .
$$

Hence $x_{0} \rightarrow F_{0}$ is a linear isometric mapping of $\mathfrak{X}$ onto a subspace of $\left(X^{+}\right)^{*}$. It remains to show that $X \subset\left(X^{+}\right)^{+}$in the above sense. This in turn requires

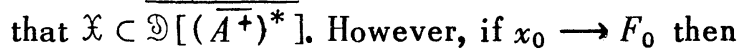

$$
\left[R^{*}\left(\lambda ; \overline{A^{+}}\right) F_{0}\right]\left(x^{+}\right)=F_{0}\left[R\left(\lambda ; \overline{A^{+}}\right) x^{+}\right]=\left[R\left(\lambda ; \overline{A^{+}}\right) x^{+}\right]\left(x_{0}\right)=x^{+}\left[R(\lambda ; \bar{A}) x_{0}\right] \text {. }
$$

Hence

$$
R(\lambda ; \bar{A}) x_{0} \rightarrow R^{*}\left(\lambda ; \overline{A^{+}}\right) F_{0} .
$$

Now

$$
\lim _{\lambda \rightarrow \infty} \lambda R(\lambda ; \bar{A}) x_{0}=x_{0}
$$

implies that

$$
\lim _{\lambda \rightarrow \infty} \lambda R^{*}\left(\lambda ; \overline{A^{+}}\right) F_{0}=F_{0} \text {; }
$$


and since

$$
R^{*}\left(\lambda ; \overline{A^{+}}\right) F_{0} \in \mathcal{D}\left[\left(\overline{A^{+}}\right)^{*}\right]
$$

it follows that $x_{0} \in \mathcal{S}\left[\left(\overline{A^{+}}\right)^{*}\right]$.

The space $\mathfrak{X}^{++}$depends only on $T^{+}(s)$ and $X^{+}$. Further, the norm in $\mathfrak{X}^{+}$is

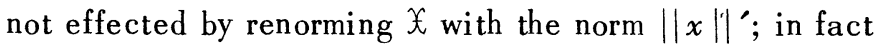

$$
\left\|x^{+}\right\|=\sup \left[\left|x^{+}(x)\right| ;\|x\|^{\prime} \leq 1, x \in \mathfrak{X}\right]
$$

Since $\mathfrak{X}$ with the norm $\|x\|^{\prime}$ is a subset of $\mathfrak{X}^{++}$, it follows that

$$
\left\|x^{+}\right\|^{\prime} \equiv \sup \left[\left|x^{++}\left(x^{+}\right)\right| ;\left\|x^{++}\right\| \leq 1, x^{++} \in X^{++}\right]=\left\|x^{+}\right\| .
$$

Thus it is only in the case of $X_{\text {and }} \mathfrak{X}^{+}$that a nonsymmetric condition between norms may arise; for all other pairs of successive adjoint spaces the norms are symmetric. Even if $\chi$ is not renormed, $X$ will be isomorphic with its image in $\mathfrak{X}^{++}$under the natural mapping.

DEFinition 3.1. We define the $(\Gamma)$-weak topology in $\chi$ in the usual way be means of the generic neighborhood

$$
N\left(x_{0} ; x_{1}^{*}, \cdots, x_{n}^{*} ; \epsilon\right) \equiv\left[x ;\left|x_{k}^{*}\left(x-x_{0}\right)\right|<\epsilon, k=1, \cdots, n\right],
$$

where the $\left(x_{1}^{*}, \cdots, x_{n}^{*}\right)$ can be any finite subset of $\Gamma$ and $\epsilon$ is an arbitrary positive number.

It is of interest to determine when, under the natural mapping, $X^{++} \mathfrak{X}^{++}$, that is, under what conditions $\mathfrak{X}$ is reflexive relative to a given semi-group of operators $[T(s)]$. Here we assume that $氏$ has been renormed with norm $\|x\|^{\prime}$. If $X$ is a reflexive in the usual sense, then the second corollary to Theorem 2.1 asserts that $\mathfrak{X}^{+}=\mathfrak{X}^{*}$, and likewise that

$$
\mathfrak{X}^{++}=\left(X^{+}\right)^{*}=\mathfrak{X}^{* *}=X \text {. }
$$

More generally, we have:

THE ORE M 3.4. Suppose that both $[T(s)]$ and $\left[T^{+}(s)\right]$ are of class $(0, A)^{*}$, and let the norm of $\mathfrak{X}$ be given by $\|x\|^{\prime}$ : A necessary and sufficient condition for $\mathfrak{X}=\mathfrak{X}^{++}$is that $R(\lambda ; \bar{A})$ be $\left(\mathfrak{X}^{+}\right)$-weakly compact.

Proof. Suppose first that $R(\lambda ; \bar{A})$ is $\left(X^{+}\right)$-weakly compact; that is, the 
image of each bounded set is contained in an $\left(\mathfrak{X}^{+}\right)$-weakly compact subset of $X_{\text {. Let }} F_{0}$ be an arbitrary element of $\left(\mathfrak{X}^{+}\right)^{*}$. Then by Helly's theorem, given a finite subset $\pi \subset \mathfrak{X}^{+}$, there exists an

$$
x_{\pi} \in \mathfrak{X},\left\|x_{\pi}\right\| \leq 2\left\|F_{0}\right\|,
$$

such that $F_{0}\left(x^{+}\right)=x^{+}\left(x_{\pi}\right)$ for all $x^{+} \in \pi$. Ordering.the $\pi^{\prime}$ s by inclusion, we easily see that they form a directed set. Consequently,

$$
\begin{aligned}
{\left[R^{*}\left(\lambda ; \overline{A^{+}}\right) F_{0}\right]\left(x^{+}\right)=F_{0}\left[R\left(\lambda ; \overline{A^{+}}\right) x^{+}\right] } & =\lim _{\pi}\left[R\left(\lambda ; \overline{A^{+}}\right) x^{+}\right]\left(x_{\pi}\right) \\
& =\lim _{\pi} x^{+}\left[R(\lambda ; \bar{A}) x_{\pi}\right] .
\end{aligned}
$$

Since the $R(\lambda ; \bar{A})$ image of any bounded set is contained in an $\left(X^{+}\right)$-weakly compact subset of $X$, it is easily shown that there exists an $x_{0} \in \mathfrak{X}$ such that

$$
\lim _{\pi} x^{+}\left[R(\lambda ; A) x_{\pi}\right]=x^{+}\left(x_{0}\right)
$$

for all $x^{+} \in \mathfrak{X}^{+}$. Thus $R^{*}\left(\lambda ; \overline{A^{+}}\right) F_{0}$ is the image of $x_{0}$ under the natural mapping; in other words, $X \supset \subseteq\left[\left(\bar{A}^{+}\right)^{*}\right]$. This together with Theorem 3.3 shows that $\mathfrak{X}=\mathfrak{X}^{++}$.

Conversely, suppose that $X=\mathfrak{X}^{++}$. Then $R^{*}\left(\lambda ; \overline{A^{+}}\right)\left[\left(X^{+}\right)^{*}\right]$ is contained in the images of $X$. Now $R^{*}\left(\lambda ; \overline{A^{+}}\right)$is continuous in the usual weak* topology of $\left(X^{+}\right)^{*}$; hence the unit sphere, which is weakly* compact, maps onto a weakly* compact subset. Now this image lies in $⿱ ㇒$ and the weak* topology in $X_{\subset}\left(X^{+}\right)^{*}$ is the same as the $\left(\mathfrak{X}^{+}\right)$-weak topology for $\chi_{\text {. Hence }} R(\lambda ; \bar{A})$, which is essentially a restriction of $R^{*}\left(\lambda ; \overline{A^{+}}\right)$, takes bounded sets into $\left(\varkappa^{+}\right)$-weakly compact subsets of $\chi$. This concludes the proof.

COR OLLARY If $R(\lambda ; \bar{A})$ is weakly compact relative to the usual weak topology of $\mathfrak{X}$, then $\mathfrak{X}=\mathfrak{X}^{++}$.

Proof. It is clear that a weakly compact subset of $\chi$ is also weakly compact relative to any weaker topology such as the $\left(X^{+}\right)$-weak topology of $X_{\text {. }}$

\section{Referenaes}

1. Leonidas Alaoglu, Weak topologies of normed linear spaces, Ann. of Math. 41 (1940), 252 - 267. 
2. Nelson Dunford, Uniformity in linear spaces, Trans. Amer. Math. Soc. 44 (1938), $305-356$.

3. William Feller, The parabolic differential equations and the associated semigroups of transformations, Ann. of Math. 55 (1952), $468-519$.

4. Semi-groups of transformations in general weak topologies, Ann. of Math. 57-58 (1953), 287-308.

5. Einar Hille, Functional analysis and semi-groups, Amer. Math. Soc. Colloquium Publ. vol. 31, New York, 1948.

6. R.S. Phillips, An inversion formula for Laplace transforms and semi-groups of linear operators, Ann. of Math. 59 (1954), 325 - 356.

UNIVERSity OF SOUThERN CALIFORNIA 



\section{PACIFIC JOURNAL OF MATHEMATICS}

\section{EDITORS}

\author{
H. L. ROYDEN \\ Stanford University \\ Stanford, California \\ E. Hewitt \\ University of Washington \\ Seattle 5 , Washington
}

R. P. Dilworth

California Institute of Technology Pasadena 4, California

A. HorN*

University of California

Los Angeles 24, California

\section{ASSOCIATE EDITORS}

\author{
H. BUSEMANN \\ HERBERT FEDERER \\ MARSHALL HALL
}

\author{
P. R. HALMOS \\ HEINZ HOPF
}

ALFRED HORN
R. D. JAMES

BORGE JESSEN

PAUL LÉVY
GEORGE PÓLYA

J. J. STOKER

KOSAKU YOSIDA

\section{SPONSORS}

UNIVERSITY OF BRITISH COLUMBIA

CALIFORNIA INSTITUTE OF TECHNOLOGY

UNIVERSITY OF CALIFORNIA, BERKELEY

UNIVERSITY OF CALIFORNIA, DAVIS

UNIVERSITY OF CALIFORNIA, LOS ANGELES

UNIVERSITY OF CALIFORNIA, SANTA BARBARA

MONTANA STATE UNIVERSITY

UNIVERSITY OF NEVADA

OREGON STATE COLLEGE

UNIVERSITY OF OREGON

UNIVERSITY OF SOUTHERN CALIFORNIA

\author{
STANFORD RESEARCH INSTITUTE \\ STANFORD UNIVERSITY \\ UNIVERSITY OF UTAH \\ WASHINGTON STATE COLLEGE \\ UNIVERSITY OF WASHINGTON
}

AMERICAN MATHEMATICAL SOCIETY HUGHES AIRCRAFT COMPANY SHELL DEVELOPMENT COMPANY

Mathematical papers intended for publication in the Pacific Journal of Mathematics should be typewritten (double spaced), and the author should keep a complete copy. Manuscripts may be sent to any of the editors. Manuscripts intended for the outgoing editors should be sent to their successors. All other communications to the editors should be addressed to the managing editor, Alfred Horn at the University of California, Los Angeles 24, California.

50 reprints of each article are furnished free of charge; additional copies may be obtained at cost in multiples of 50 .

The Pacific Journal of Mathematics is published quarterly, in March, June, September, and December. The price per volume (4 numbers) is $\$ 12.00$; single issues, $\$ 3.50$. Back numbers are available. Special price to individual faculty members of supporting institutions and to individual members of the American Mathematical Society: $\$ 4.00$ per volume; single issues, $\$ 1.25$.

Subscriptions, orders for back numbers, and changes of address should be sent to Pacific Journal of Mathematics, c/o University of California Press, Berkeley 4, California.

Printed at Kokusai Bunken Insatsusha (International Academic Printing Co., Ltd.), No. 10, 1-chome, Fujimi-cho, Chiyoda-ku, Tokyo, Japan.

* During the absence of E. G. Straus.

PUBLISHED BY PACIFIC JOURNAL OF MATHEMATICS, A NON-PROFIT CORPORATION COPYRIGHT 1955 BY PACIFIC JOURNAL OF MATHEMATICS 


\section{Pacific Journal of Mathematics}

\section{Vol. 5, No. $2 \quad$ October, 1955}

Leonard M. Blumenthal, An extension of a theorem of Jordan and von

Neumann ........................................ 161

L. Carlitz, Note on the multiplication formulas for the Jacobi elliptic functions.......................................... 169

L. Carlitz, The number of solutions of certain types of equations in a finite

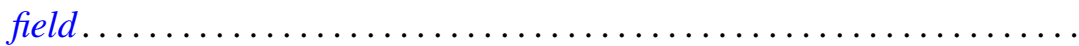

George Bernard Dantzig, Alexander Orden and Philip Wolfe, The generalized simplex method for minimizing a linear form under linear

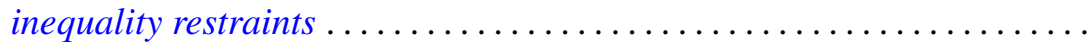

Arthur Pentland Dempster and Seymour Schuster, Constructions for poles and polars in n-dimensions . . . . . . . . . . . . . . . . . . . . 197

Franklin Haimo, Power-type endomorphisms of some class 2 groups ...... 201

Lloyd Kenneth Jackson, On generalized subharmonic functions ......... 215

Samuel Karlin, On the renewal equation ...................... 229

Frank R. Olson, Some determinants involving Bernoulli and Euler numbers of higher order................................ 259

R. S. Phillips, The adjoint semi-group ........................ 269

Alfred Tarski, A lattice-theoretical fixpoint theorem and its applications ... 285

Anne C. Davis, A characterization of complete lattices .............. 311 